\title{
The Role of Discrete Capillary Rings in Mass Transfer From the Surface of a Drying Capillary Porous Medium
}

\author{
Hafiz Tariq Mahmood ${ }^{1} \cdot$ Evangelos Tsotsas $^{1} \cdot$ Abdolreza Kharaghani $^{1}$ (D)
}

Received: 15 January 2021 / Accepted: 8 June 2021 / Published online: 18 June 2021

(c) The Author(s) 2021

\begin{abstract}
The mass exchange between the surface of a model capillary porous medium and the adjacent gas-side boundary layer is studied in the limiting condition of isothermal, slow drying. In order to quantify the role and significance of liquid films in the mass exchange process, three-dimensional pore network Monte Carlo simulations are carried out systematically in the presence and absence of discrete capillary rings. The pore network simulations performed with capillary rings show a noticeable delay in transition from the capillary-supported regime to the diffusion-controlled regime. These simulation results differ significantly from the predictions of classical pore network models without liquid films, and they appear to be more consistent with the experiments conducted with real porous systems. As compared to classical pore network models, the pore network model with rings seems to predict favorably the spatiotemporal evolution of wet and dry patches at the medium surface as well as of their relative contributions to the net mass exchange rate. This is apparent when the analytical solution of the commonly used Schlünder's model is examined against the numerical simulations conducted using classical and ring pore network models.
\end{abstract}

Keywords Capillary rings · Drying · Discrete liquid films · Pore network modeling · Surface phenomena

\section{Introduction}

Drying is a mass transfer process that involves successive removal of a volatile liquid by evaporation from a moist porous medium. Despite the fact that this process is central to many natural systems and a major unit operation in industrial sectors, a quantitative description of key mass transport mechanisms such as liquid film flow has not yet fully provided. Traditional approach treats a partially saturated porous medium as a spurious continuum for which effective transport parameters are expressed as a function of average liquid content. Though several models have been developed within the framework of this approach (Philip and Vries 1957; Luikov, 1966; Whitaker, 1998), they all have

Abdolreza Kharaghani

abdolreza.kharaghani@ovgu.de

1 Thermal Process Engineering, Otto von Guericke University Magdeburg, P.O. 4120,

39016 Magdeburg, Germany 
limited predictive capacity in relation to transition from the capillary-supported regime to the diffusion-controlled regime. Conversely, pore network models offer a discrete approach in which transport phenomena are described at the level of single pores which are interlinked. Originally, pore network models are used in hydrogeology (Sahimi et al. 1986) and petroleum engineering (Lenormand et al. 1988; Dillard and Blunt, 2000) to study groundwater flow and secondary oil recovery. In early nineties, Daian and Saliba (1991) and Nowicki et al. (1992) coined the idea of discrete modeling of capillary porous materials drying. Later on, Prat (1993) proposed a first pore network drying model by using concepts of invasion percolation (Wilkinson and Willemsen, 1983) and calculation of diffusive vapor transport. Since this pioneering work, various researchers have incorporated pore-scale physical effects, either individually or combined, into this model and studied their impact on the drying characteristics of capillary porous media. Examples include gravity (Laurindo and Prat 1996; Yiotis et al. 2012), liquid viscosity (Metzger et al. 2007a), gas-side boundary layer (Yiotis et al. 2007), heat transfer (Surasani et al. 2008), capillary valve effect (Wu et al. 2016, 2017) and damaging effects of capillary forces (Kharaghani et al. 2012). The pore-scale physical effect in connection with the present work is film flow which has also been studied numerically and experimentally in systems ranging from a single capillary tube (Chauvet et al. 2009, 2010), two-dimensional pore networks (Laurindo and Prat, 1998; Yiotis et al. 2004), to quasi-two-dimensional micromodels (Vorhauer et al. 2015; Chen et al. 2017, 2018), as well as to much more complex systems such as three-dimensional (3D) particle packings (Wang et al. 2012; Kharaghani et al. 2021). In those model porous systems, liquid films have the form of either corner films or capillary bridges. Corner liquid films appear in the margins of non-circular pores even after the core of such pores has been displaced by gas during evaporation. The impact and dynamics of corner films have been studied using the continuous film pore network model. This model highly overestimates the extent of the corner film region, and thus, the predicted overall phase distribution appears to be unrealistic. Capillary bridges form at the contact between particles in packed beds, for example. The influence of capillary bridges (or capillary rings) on drying kinetics has been investigated using the capillary ring pore network model. In contrast to the continuous film model, the ring model can lead to phase distributions strongly resembling measured distributions (e.g., see, Vorhauer et al. (2015) and references therein). Common to all these works has been that liquid films play a crucial role in intraparticle mass transport, that is mass transport within drying capillary porous media. However, the role and significance of liquid films in relation to the mass transport at the interface between the porous medium surface and the adjacent bulk air have not been widely studied in literature, neither experimentally nor numerically.

Linking the surface mass transfer attributes with the adjacent bulk air has been considered as a specific research topic by Schlünder (1988, 2004). His main motivation was essentially to explain why the evaporation rate from the surface of a partially saturated porous medium is nearly identical to that of a fully saturated surface. In other words, a simple mathematical description of a long constant rate period (CRP) in drying kinetic curves determined experimentally was aimed. In order to derive an analytical model, Schlünder made four assumptions: (i) Only wet patches at the surface contribute to the net evaporation rate, (ii) wet patches are hemispherical droplets with uniform radius $\left(r_{t}\right)$ which are evenly placed inside squares of size $(21 \times 21)$, (iii) the liquid vapor generated by evaporation is transported by equimolar diffusion from the medium surface to the bulk air and (iv) wet patches have radii much smaller than the thickness of the external boundary layer $(\delta)$. On the basis of these assumptions, Schlünder derived the following correlation: 


$$
\frac{J}{J_{\max }}=\frac{1}{1+\frac{2 r_{t}}{\pi} \sqrt{\frac{\pi}{4 \theta}}\left(\sqrt{\frac{\pi}{4 \theta}}-1\right)},
$$

where $\mathbf{J}$ denotes the evaporation rate, $J_{\max }$ the initial evaporation rate when the surface is fully wet and $\theta=\frac{\pi r_{t}^{2}}{(2 l)^{2}}$ the wetted surface area.

The surface attributes during the drying process are not as simple as "seen" and formulated by Schlünder. The research conducted by classical 3D pore network model (CPNM) simulations shows that instead of only wet patches (represented by liquid-filled surface pores in PNM) both wet patches and dry patches (represented by empty surface pores in PNM) contribute to the mass exchange at the medium surface (Moghaddam et al. 2018). The study conducted by Talbi and Prat (2019) clearly shows that the size distribution of wet patches and the shape of droplets impact the drying characteristics at the surface. Details of Schlünder's model in connection to closure relationship and CPNMs have thoroughly been described in previous works (e.g., Lehmann and Or, 2013; Moghaddam et al. 2018; Talbi and Prat, 2019; Lu et al. 2021). While these works contributed to a much better understanding of the mass transfer at the surface, they disregarded the impact of the liquid films developing in geometrical singularities of the pore space during drying.

In this work, the extent over which capillary rings can impact the mass exchange between the porous medium surface and the bulk air is studied. For this purpose, numerical simulations of a drying capillary porous medium are carried out using the CPNM and ring pore network model (RPNM). The results obtained from these two models are compared against the model proposed by Schlünder.

The outline of this paper is as follows: In Sect. 2, a brief description of the RPNM algorithm is presented. In Sect. 3, the results in terms of drying kinetics, liquid phase distribution, and vapor pressure field at the medium surface are discussed. Summary of major findings and a future research direction are outlined in Sect. 4.

\section{Ring Pore Network Model}

The ring pore network model (RPNM) presented here is reported in our earlier work, in which we studied the impact of capillary rings on intraparticle mass transport during drying of quasi-two-dimensional networks (Vorhauer et al. 2015) and of three-dimensional particle packings (Kharaghani et al. 2021). In this model, the void space of a capillary porous medium is approximated by a network of throats (primary capillary structures) and rings (secondary capillary structures) (Fig. 1). Throats are cylindrical tubes with uniform length and radii generated randomly. Rings are concave cylinders with identical volume which represent discrete liquid films. In addition to throats and rings, pores without volume exist which act as numerical nodes between throats. The state variables such as liquid or vapor pressure are assumed at pores.

The saturation state of throats, rings and pores is specified prior to solving mass balances. Throats and rings can be full, partially filled or empty as shown in Fig. 1. The state of pores is defined as follows: liquid pore when surrounded by liquid throats and rings, gas pore at the equilibrium vapor pressure $\left(P_{\mathrm{v}, \mathrm{eq}}\right)$ when at least one neighboring element is liquid or gas pore at vapor partial pressure $\left(P_{\mathrm{v}}\right)$ when all neighboring throats and rings are occupied by the gas phase. Menisci in throats and rings depend upon gaseous state of their neighboring pores and throats. The gas pores generate menisci in neighboring throats, 


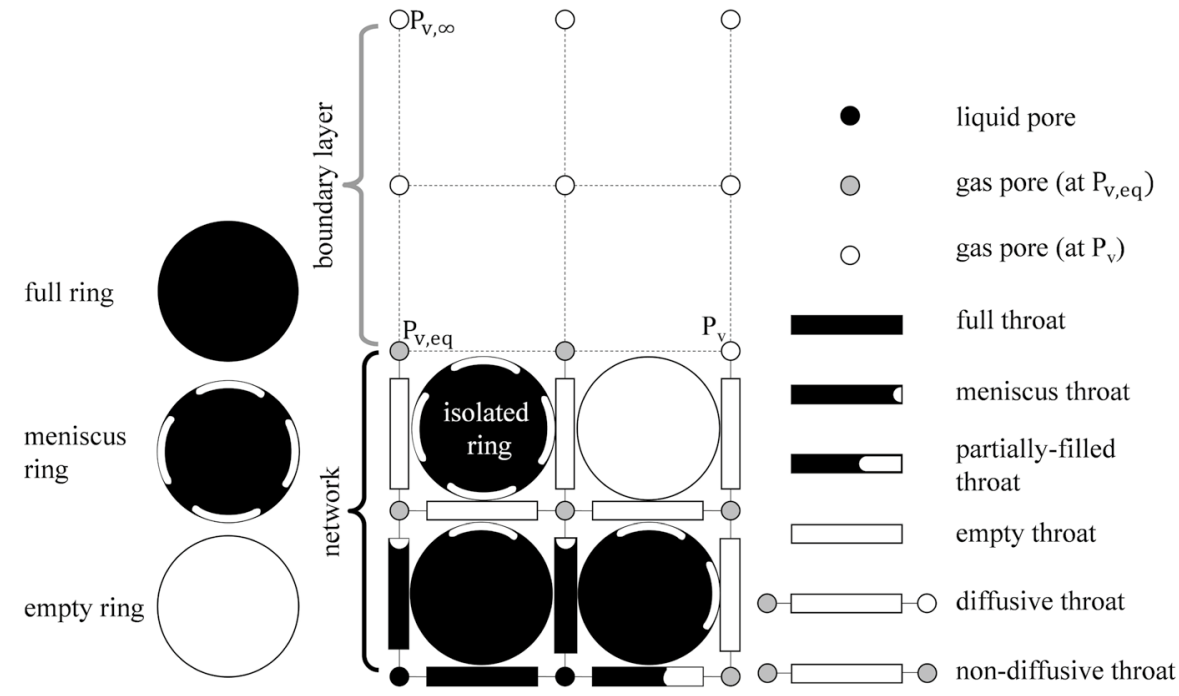

Fig. 1 A partially saturated ring pore network with the gas-side boundary layer illustrating saturation states of throats, rings and pores

while empty throats generate menisci in neighboring rings. The menisci throats participate in capillary pumping, and they can lead to a pressure drop, while the menisci rings do not participate in capillary pumping and maintain additional pathway for fluid transport. Throats surrounded with the gas pores are single throats in CPNM; however, this limitation of isolation no longer exists in RPNM. Rings surrounded by all gas throats are single rings, which are similar to single throats of CPNM. Moreover, gas throats are categorized as nondiffusive throats with both pores at $P_{\mathrm{v}, \mathrm{eq}}$ or as diffusive throats with one or both pores at $P_{\mathrm{v}}$.

Based on the saturation state of throats and rings, four zones are distinguished as: liquid zone (full, partially filled throats and full rings), film zone (fully or partially filled meniscus rings and empty rings neighboring to non-diffusive throats), non-diffusive gas zone (nondiffusive throats) and dry zone (diffusive throats and their neighboring empty rings). The cumulative volume fraction of these zones stands unity at any given saturation of the network during drying (see Sect. 3).

A small 3D cubic ring pore network is depicted in Fig. 2, where the gas-side boundary layer is shown in black dashed lines. Cylinders on each face of each cube illustrate rings. Edge and vertex of each cube illustrate throats and pores, respectively. The liquid evaporates from the top side of the network, and periodic boundary conditions are imposed in horizontal directions.

The parameters used for both PNM simulations are presented in Table 1. The boundary layer is embodied by adding 10 nodes in vertical direction with the same cross section of the network $(25 \times 25)$. The results are averaged over 15 realizations for each model. The drying process is limited to isothermal capillary dominated regime for non-adsorbed water. Vapor diffusion in the film and non-diffusive gas zones is suppressed. Gas pores at equilibrium vapor pressure and vapor pressure of dry air at uppermost layer of pores of the external boundary layer $\left(P_{\mathrm{v}, \infty}=0\right)$ provides boundary conditions. The diffusive transport in the boundary layer is considered. Quasi-steady vapor flow rate through a throat of nodes $\mathrm{i}$ and $\mathrm{j}$ is computed by 
Fig. 2 Representation of a 3D ring pore network with the gasside boundary layer attached to the network surface

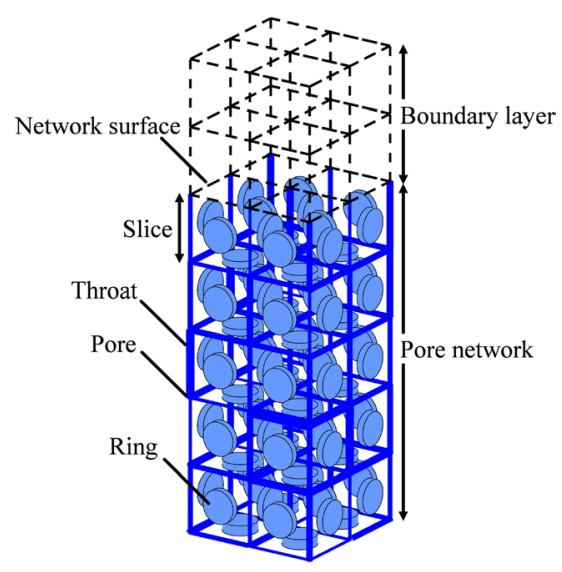

Table 1 Parameters used for CPNM and RPNM drying simulations

\begin{tabular}{lll}
\hline Parameter & CPNM & RPNM \\
\hline Pore network size & $25 \times 25 \times 51$ nodes & $25 \times 25 \times 51$ nodes \\
Boundary layer size & $25 \times 25 \times 10$ nodes & $25 \times 25 \times 10$ nodes \\
Mean throat radius $(\mu \mathrm{m})$ & 207 & 207 \\
Standard deviation in throat radii $(\mu \mathrm{m})$ & 20.7 & 20.7 \\
Throat length $(\mu \mathrm{m})$ & 1000 & 1000 \\
Ring radius $(\mu \mathrm{m})$ & - & 252 \\
Ring thickness $(\mu \mathrm{m})$ & - & 137.1 \\
Temperature $\left({ }^{\circ} \mathrm{C}\right)$ & 20 & 20 \\
Gas pressure $(\operatorname{atm})$ & 1 & 1 \\
Network porosity $(-)$ & 0.44 & 0.49 \\
Top network surface porosity $(-)$ & 0.15 & 0.18 \\
\hline
\end{tabular}

$$
\sum_{\mathrm{j}} \dot{M}_{\mathrm{v}, \mathrm{jj}}=\sum_{\mathrm{j}} A_{\mathrm{ij}} \frac{D_{\mathrm{va}}}{L_{\mathrm{ij}}} \frac{\tilde{M}_{\mathrm{v}} P}{\tilde{R} T} \ln \left(\frac{P-P_{\mathrm{v}, \mathrm{i}}}{P-P_{\mathrm{v}, \mathrm{j}}}\right),
$$

where $A_{\mathrm{ij}}$ is the exchange area ( $L^{2}$ for boundary layer and $\pi \mathrm{r}_{\mathrm{ij}}^{2}$ for network), $L_{\mathrm{ij}}$ the distance between two nodes, $P_{\mathrm{v}, \mathrm{i}}$ the vapor pressure and $\dot{M}_{\mathrm{v}, \mathrm{ij}}$ the vapor mass flow rate, T the air temperature, $\tilde{R}$ the universal gas constant, $P$ the atmospheric pressure and $D_{\mathrm{va}}$ the binary diffusion coefficient, $\tilde{M}_{\mathrm{v}}$ the molar mass of vapor. Each neighbor meniscus element of the diffusive pore $\mathrm{i}$ (provide diffusion) contributes to the global evaporation rates according to its cross section area, calculated as

$$
\begin{aligned}
& \dot{M}_{\mathrm{e}, \mathrm{ik}}=\frac{A_{\mathrm{ik}}}{A_{\mathrm{t}, \mathrm{i}}} \sum_{\mathrm{m}} \dot{M}_{\mathrm{v}, \mathrm{im}}, \\
& \dot{M}_{\mathrm{e}, \mathrm{ir}}=\frac{A_{\mathrm{ir}}}{A_{\mathrm{t}, \mathrm{i}}} \sum_{\mathrm{m}} \dot{M}_{\mathrm{v}, \mathrm{im}},
\end{aligned}
$$


where $\mathrm{A}_{\mathrm{ik}}$ is the cross section area of the meniscus throat of nodes $\mathrm{i}$ and $\mathrm{k}, A_{\mathrm{ir}}$ is the evaporating area of the liquid ring $\mathrm{r}$ that is neighbor to pore $\mathrm{i}$ (half, three quarters and full of lateral area $(2 \pi \mathrm{rh})$ based on one, two or more than two menisci in liquid ring $\mathrm{r}$ ), $A_{\text {tot, } \mathrm{i}}=\sum_{\mathrm{k}} A_{\mathrm{k}}+\sum_{\mathrm{r}} A_{\mathrm{r}}$ is the total area of all neighbor meniscus throats and neighbor liquid rings of pore i, $\sum_{\mathrm{m}}^{\mathrm{r}} \dot{M}_{\mathrm{v}, \mathrm{im}}$ is the total vapor flow rate away from the gas pore $\mathrm{i}$ through the neighboring empty throats.

Labeling of elements is important to track the time evolution of the liquid and gas phases (Metzger et al. 2007b). Initially, all elements belong to one cluster. The principle of ring pore network labeling is if two throats are bridged by a liquid ring, they belong to the same cluster based on modified Hoshen-Kopelman (HK) algorithm. The labeling process starts from emptied element and restricted to the cluster of that emptied element. Salient components of the RPNM drying algorithm have been explained in detail in our earlier work (Kharaghani et al. 2021). The overall drying algorithm is summarized in a flowchart shown in Fig. 3.

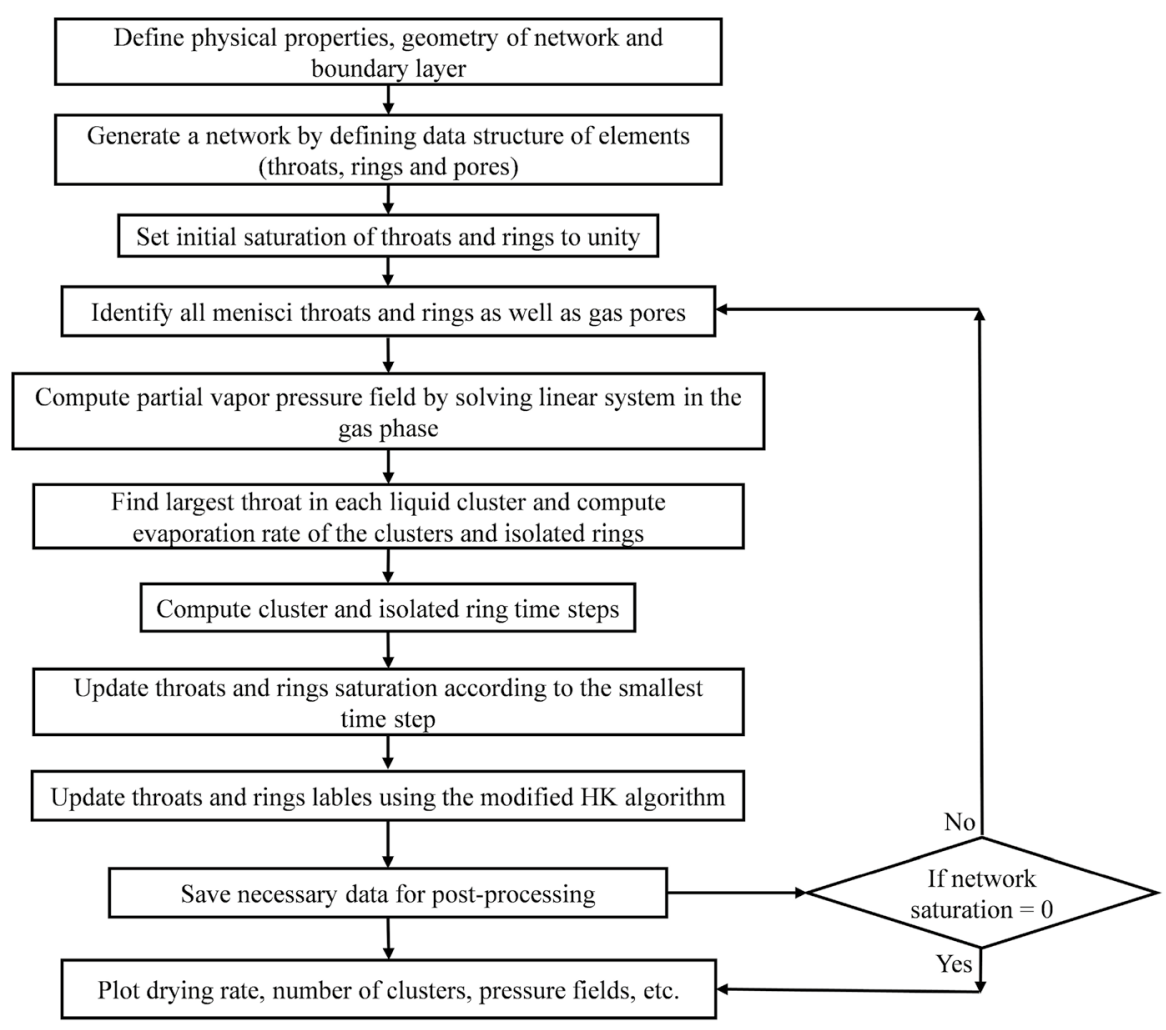

Fig. 3 Flowchart of the ring pore network drying algorithm 


\section{Results and Discussion}

The normalized evaporation rate and surface saturation obtained from both pore network models, i.e., CPNM and RPNM, under identical initial and boundary conditions are shown in Fig. 4. Both models are capable of predicting three main periods of drying of capillary porous media, but with different extent. During constant rate period (CRP), the evaporation rate decreases slightly in the CPNM. The drop from the initial evaporation rate termed as initial transition period has also been observed in previous classical pore network simulations (Moghaddam et al. 2017 and 2018). In these studies, the drop was commonly deduced to occur due to the invasion of surface pores from the beginning of the drying process until the gas phase breakthrough, i.e., until the gas phase reaches the network bottom for the first time. Interestingly, even though the surface pores are invaded by the gas phase and the breakthrough predicted by both CPNM and RPNM occurs more or less at the same time (see circles in Fig. 4), the drop is completely circumvented and the evaporation rate remains at the highest initial value for a significant period of drying in RPNM. In order to find out reasons underlying this observation, the saturation of the network surface is calculated for both types of pore network. The surface in RPNM is defined as all vertical throats and rings at the interface between the network and bulk air, whereas only vertical throats at the interface describe the surface in CPNM. A sharp drop in the saturation of the network surface is readily notable in both pore networks, but with magnitudes that differ by a factor of more than two (see Fig. 4). At first sight it may appear that this difference attributes to the decrease in evaporation rate during the initial transition period. If this was the only reason, one would also expect a decrease in the evaporation rate of RPNM, though to a smaller extent. We will get back to this point later on in this section.

When CRP ends, the evaporation rate drops significantly and the falling rate period (FRP), often called the diffusion-controlled regime, onsets. At some point during the CRP/ FRP transition, the liquid phase does not spread the whole height of the network (irreducible saturation) as indicated by squares in Fig. 4. Compared to CPNM (also, see, Yiotis et al. 2006; Moghaddam et al. 2017), the irreducible saturation of RPNM is very low,

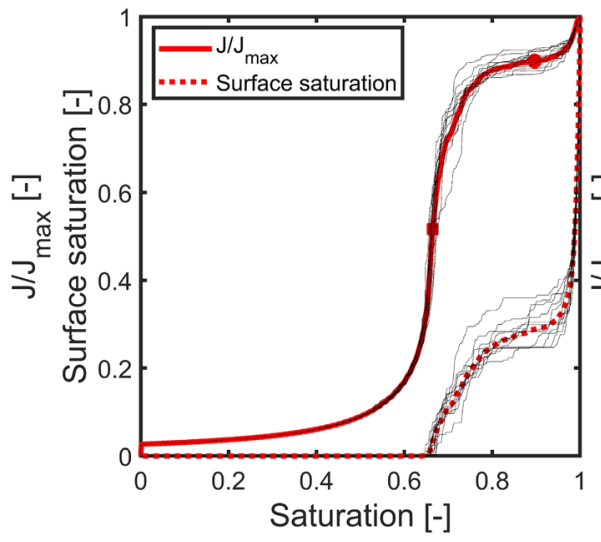

(a)

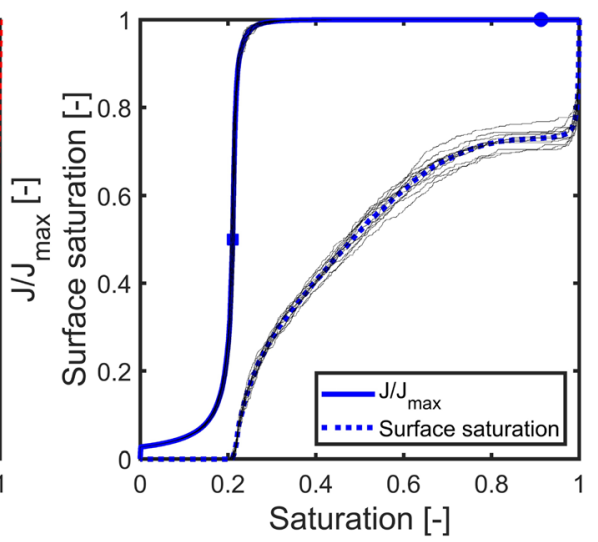

(b)

Fig. 4 The normalized evaporation rate and surface saturation as a function of network saturation predicted by a CPNM and b RPNM. All 15 realizations (thin lines) and the arithmetic mean (thick lines) are shown. The instants at breakthrough and irreducible saturation are indicated by circles and squares, respectively 
similarly as in experiments (Faure and Coussot, 2010). The decrease in irreducible saturation is caused by the liquid rings within the network. The inclusion of this secondary capillary structure in the RPNM promotes spanning of the main liquid cluster and also increases the coordination number of pores from 6 neighboring throats in CPNM to additional 12 neighboring rings in RPNM.

Coming back to the question why the immediate drop in surface saturation cannot reduce the evaporation rate in the RPNM. In other words, why the initial transition period is completely bypassed in the RPNM. To answer this question, the transport zones defined in Sect. 2 are computed using both types of pore networks and the respective simulation results are visualized in Fig. 5. As can clearly be seen, the role of liquid rings in distribution

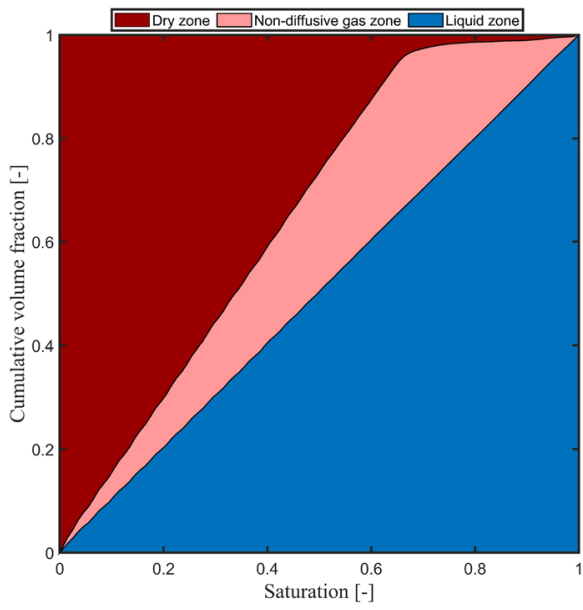

(a)

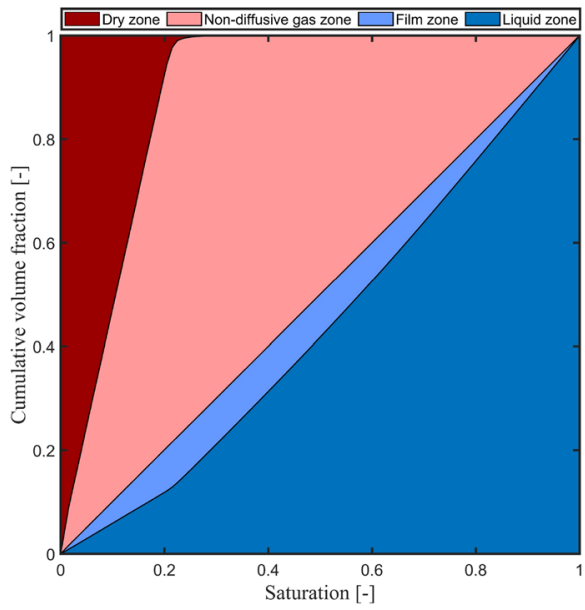

(c)

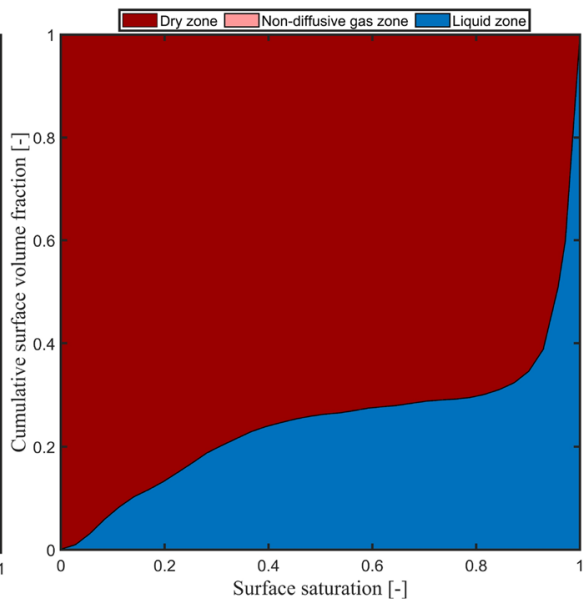

(b)

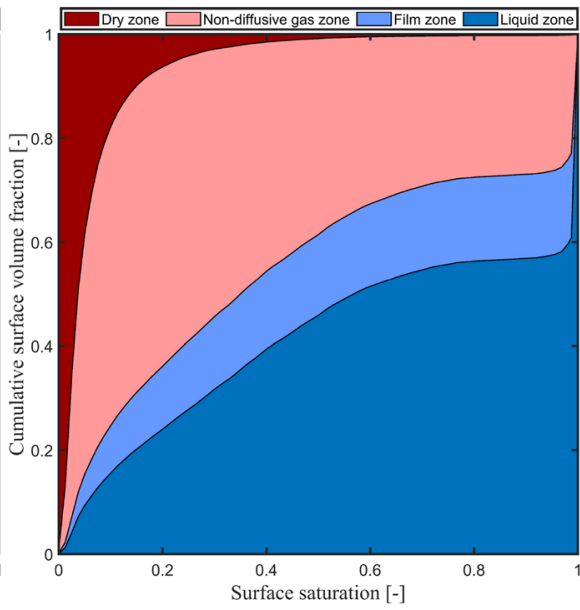

(d)

Fig. 5 Cumulative (surface) volume fractions of mass transport zones as a function of network/surface saturation predicted by $\operatorname{CPNM}(\mathbf{a}, \mathbf{b})$ and by $\operatorname{RPNM}(\mathbf{c}, \mathbf{d})$. The transport zones developed inside the pore networks $(\mathbf{a}, \mathbf{c})$ and at their respective surfaces $(\mathbf{b}, \mathbf{d})$. The data have been averaged over 15 realizations 
of the liquid and gas phases is remarkable. Though the rings contribute only $7.7 \%$ to the total liquid volume, they can assure liquid transport from the network inside toward its surface, hence enhancing and prolonging the surface wetness. The zones occupied by the gas phase, i.e., non-diffusive gas zone and dry (or diffusive gas) zone, are distinguished based on their resistance for vapor transport. Vapor diffusion is fully suppressed in the nondiffusive gas zone, whereas the dry zone poses resistance to vapor transport. Right from the beginning of drying the non-diffusive gas zone develops and its relative contribution to the total volume of zones increases during CRP, thereby an initial transition period cannot be developed in the RPNM.

Another interesting piece of information that can be inferred from Fig. 5 is the transition from the FRP to the RFP. This transition point occurs when the extent of the dry zone across the network becomes the largest (cf. Figures 4 and 5).

The impact of rings on the time evolution of the vapor pressure field at the network surface is shown in Fig. 6. During the initial transition period of the CPNM, the quick removal of liquid from the surface reduces the vapor pressure at the entrance of surface pores from the equilibrium vapor pressure $\left(P_{\mathrm{v}, \mathrm{eq}}\right)$ to lower values. Thus, the fraction of the surface pores at vapor partial pressure $\left(P_{\mathrm{v}}\right)$, which are not connected to the liquid phase, starts increasing progressively from the beginning of the drying process until complete drying of the network. When almost half of the network surface has been dried, the number of surface pores at $P_{\mathrm{v}}$ becomes dominant, while all the surface pores in the ring pore network remain at equilibrium vapor pressure during this period. This transition point occurs when only a small amount of the total liquid $(\sim 2 \%)$ is evaporated from the classical pore network. However, the transition point in RPNM is reached when an enormous amount of the total liquid ( 75\%) has been evaporated (cf. Figures 6 and 4 ).

The influence of rings on the evolution of the liquid structures (isolated elements and clusters) inside the network and at the surface is presented in Fig. 7. As can be seen, the number of single throats and clusters at the surface in CPNM increases significantly right from the beginning of the drying process. This behavior again explains the initial transition period in CPNM. After this period, the liquid structures at the surface develop with a similar trend in both models with an exception of delayed disintegration in RPNM (Fig. 7a and $b$ ). Inside the network, the disintegration of the liquid structure occurs at a later stage of CRP in RPNM, while it starts from the beginning in CPNM. In both networks, the maximum number of liquid structures exists near the end of CRP (Fig. 7c and d).

A detailed analysis of the evolution of liquid structures inside the network based on the position and the main liquid cluster provides interesting insight into the phenomena

Fig. 6 Fraction of surface pores at equilibrium vapor pressure $\left(P_{\mathrm{v}, \mathrm{eq}}\right)$ and pores at averaged vapor partial pressure $\left(P_{\mathrm{v}}\right)$ as a function of surface saturation, averaged over 15 realizations

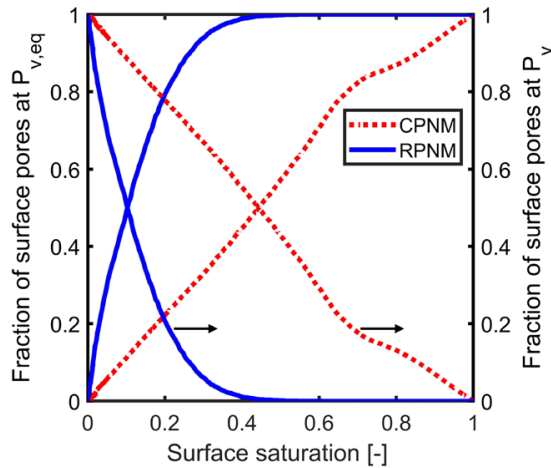




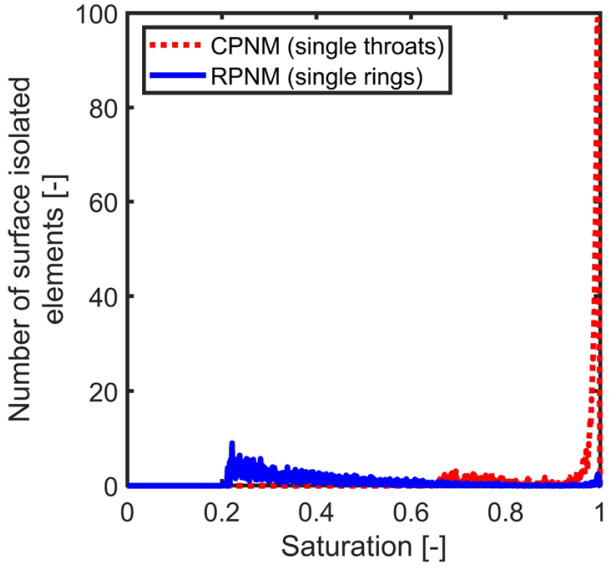

(a)

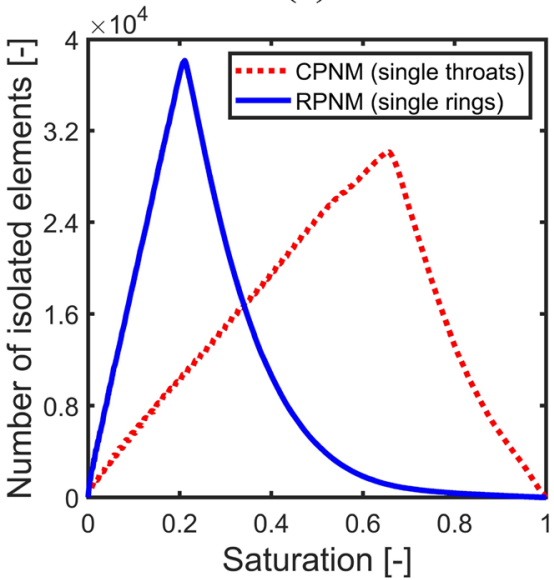

(c)

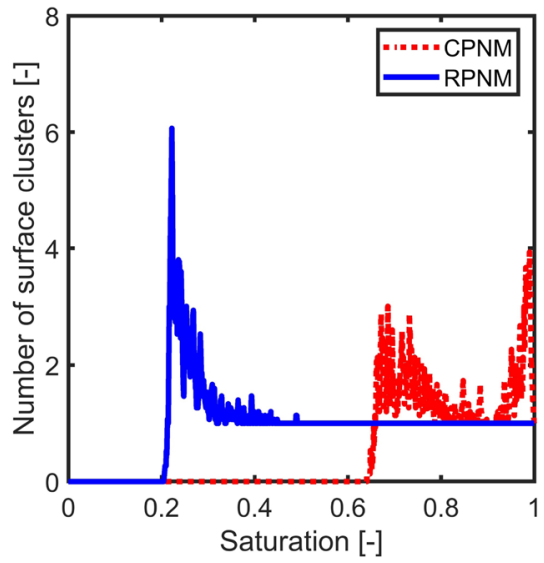

(b)

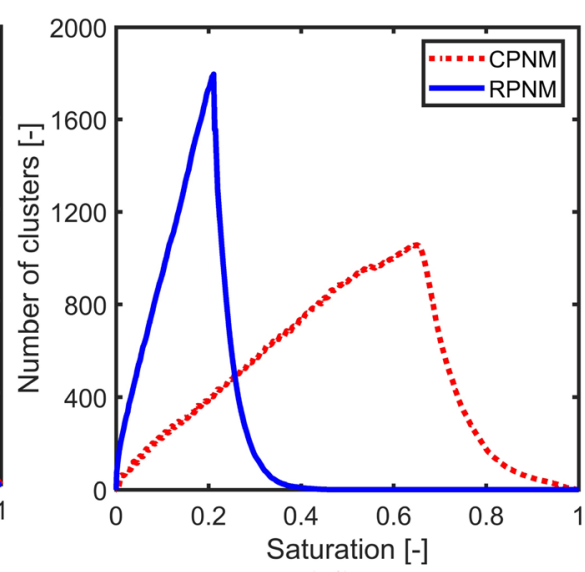

(d)

Fig. 7 For both CPNM and RPNM: a number of isolated elements at the network surface, $\mathbf{b}$ number of clusters connected to the network surface, $\mathbf{c}$ number of isolated elements in the network and $\mathbf{d}$ number of clusters in the network as a function of network saturation, averaged over 15 realizations

that occur during drying. As shown in Fig. 8, the pore network is divided into top and bottom regions in the direction of height based on proximity to the open side of the network and the opposite sealed side of the network. During CRP, the evolution of liquid structures in both regions and models is similar except an initial higher growth of the disintegration of the liquid in the top region of CPNM. Afterward, this conduct becomes contradictory. The liquid structures in the top region of the network decline, while they become almost stable in the bottom region for considerable duration in both models. This stability in the bottom region is due to increase in number of small clusters and the liquid removal from the top region only, as explained later. Apparently, duration of this stable state is longer in CPNM; however, this duration is approximately similar relative to duration of RFP in both networks. The number of clusters spreading over both regions is very small in both models and not plotted in Fig. 8. 


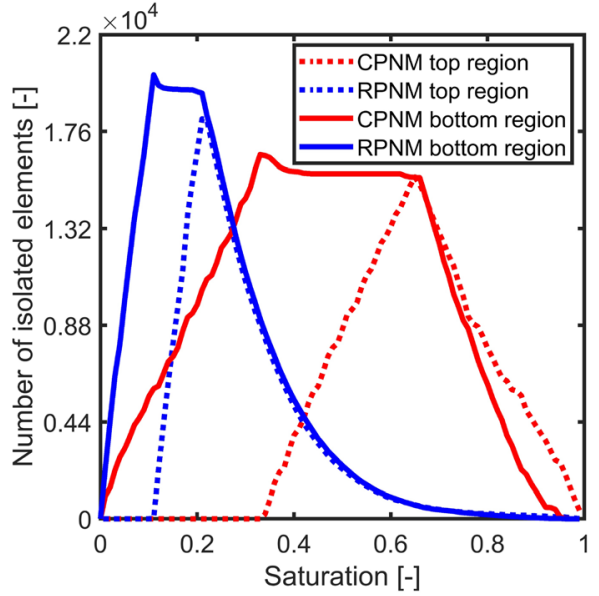

(a)

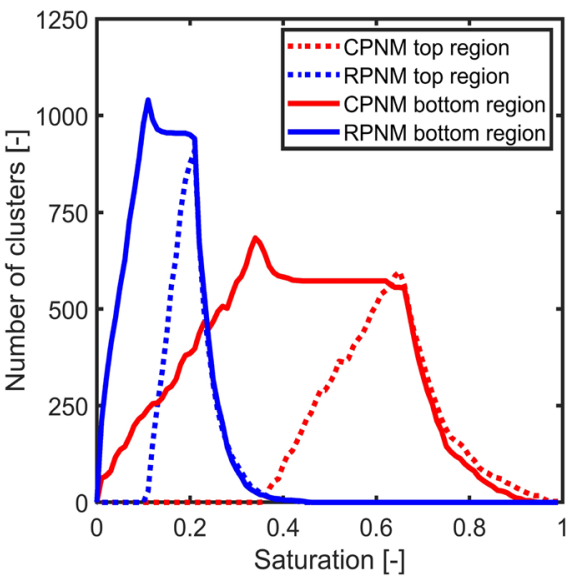

(b)

Fig. 8 Number of isolated elements (a) and clusters (b) as a function of network saturation, obtained for a single realization

Figure 9 reveals size of clusters especially the main cluster during drying. Almost all liquid belongs to the main cluster during CRP in RPNM (Fig. 9a). The relative volume of the main cluster to total liquid volume in RPNM is close to experimentally measured value (Kharaghani et al. 2021). The abrupt drop in the volume of the main cluster illustrates a high disintegration of the liquid phase inside the network and triggers CRP/FRP transition in both models. Size of other clusters is minuscule relative to the main cluster in both networks; however, a significant amount of volume belongs to the isolated

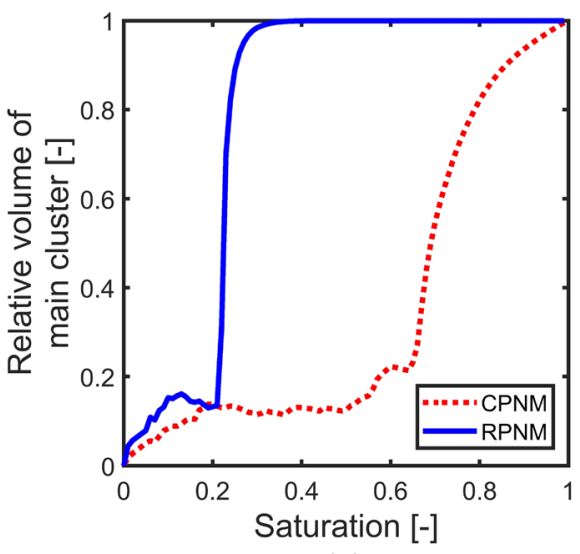

(a)

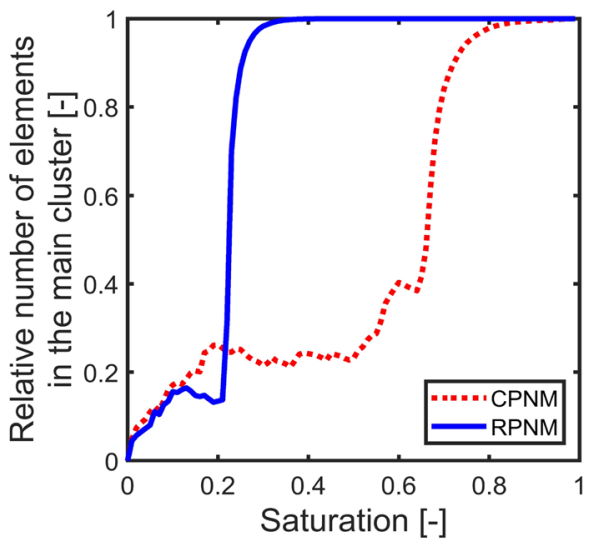

(b)

Fig. 9 a Relative volume of the main liquid cluster to the total liquid volume and $\mathbf{b}$ relative number of elements in the main liquid cluster to the total liquid clusters (excluding isolated elements) as a function of network saturation, averaged over 15 realizations 
elements in CPNM during CRP as can be concluded from disparity between Figs. 9a and $b$. After FRP, size of all clusters is small and comparable.

The network saturation reaches the irreducible saturation during the CRP/FRP transition in both models. The impact of the irreducible saturation is shown in Fig. 10. Each slice of the ring pore network contains horizontal and vertical planes of throats and rings (see Fig. 2), while it consists of only throats in the classical pore network. As pointed out previously, the process of liquid percolation from the bottom slice to the surface slice is terminated after the irreducible saturation. This is obvious from the profiles of sliceaveraged saturations (cf. Figure 10). After 0.66 network saturation in CPNM, saturation of the bottom slice remains nearly unchanged, while in RPNM saturation of the bottom slice decreases until 0.21 . These profiles are spatially uniform along the height during CRP in both models. A sharp drop in profiles of saturation near the surface of evaporation (top edge effect) is not dominant in RPNM as it is in CPNM because of gradual decrease in surface saturation and absence of initial period (see Fig. 10).

As discussed above, the drying process can be described by the liquid cluster formation inside the network and at the network surface. The residence time of the cluster depends upon the position and size of the cluster. A cluster will stay longer if it is large and stay farther away from the network surface. Figure 11 shows the top view of the network where throats are represented by circles and rings by rectangles. Different colors have been assigned to different clusters. If two elements are not connected at the surface but they are connected inside the network, they belong to the same cluster. Diffusive empty throats and empty rings are shown in white, non-diffusive gas throats in off-white, and isolated elements in red. The biggest cluster at the surface is visualized in blue. The total number of throats at the surface is 625 in both models. The surface of RPNM contains additional 1250 rings with an attribution of only 3\% additional volume. The RPNM and CPNM are visualized side by side for comparison. In addition to values of the surface saturation, values of the network saturation are shown.

Saturation of the surface decreases rapidly before the breakthrough occurs in both models. After breakthrough, saturations of both the surface and network decrease in RPNM, while in case of CPNM this trend is different. For surface saturation $0.90-0.60$, the

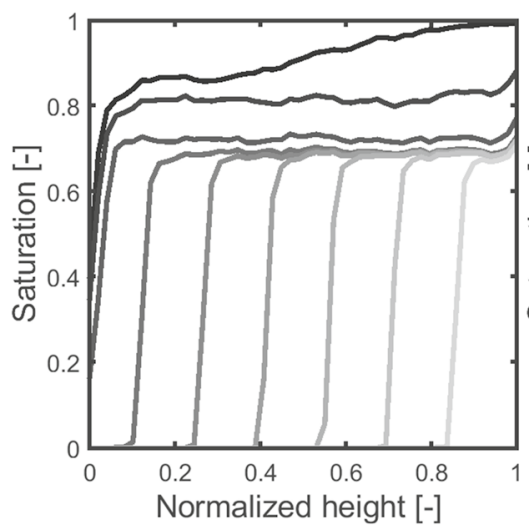

(a)

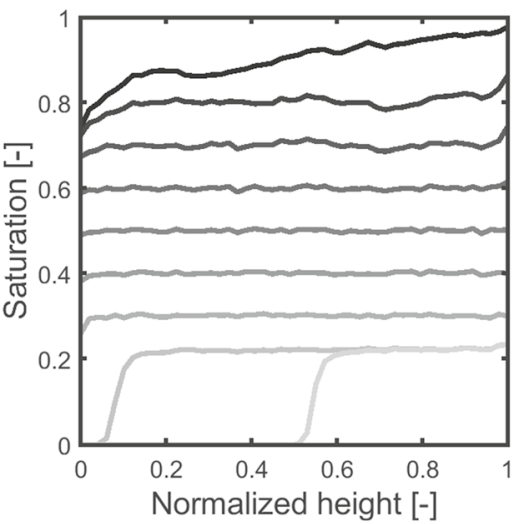

(b)

Fig. 10 Profiles of slice-averaged saturations for: a CPNM and b RPNM, averaged over 15 realizations. The network top surface is at 0 and bottom of the network is at 1 


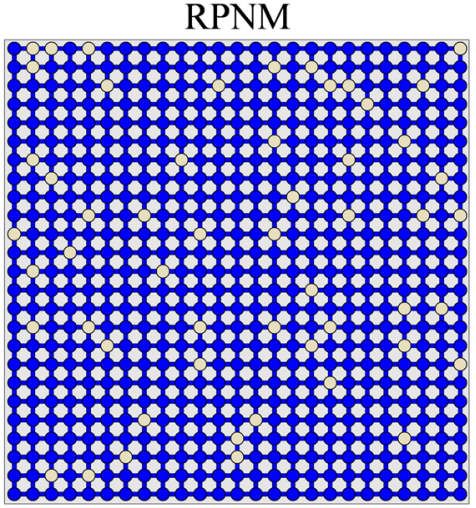

$0.90,0.99$

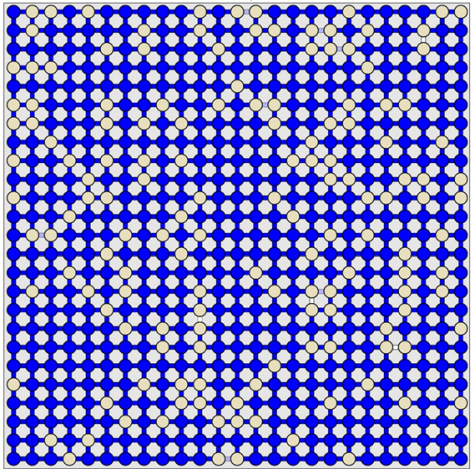

$0.75,0.96$

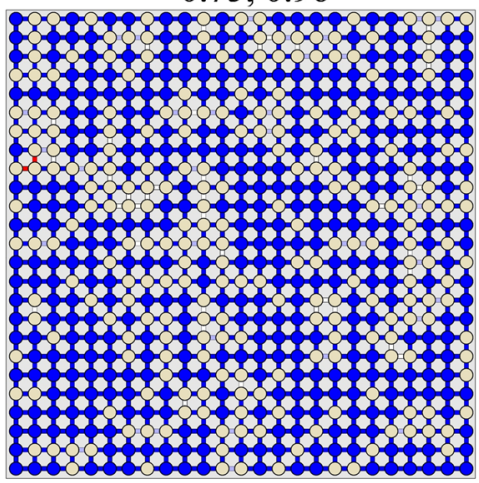

$$
0.60,0.58
$$

CPNM

0000000000000000000000000

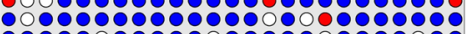

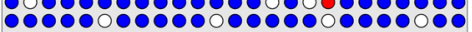


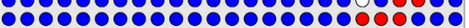
(1)    

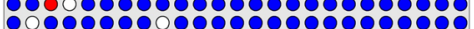
-

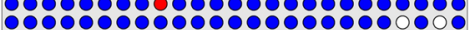

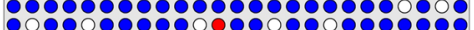

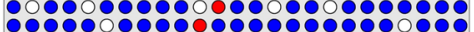

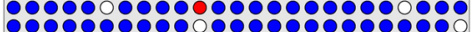

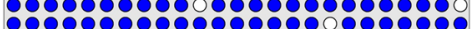

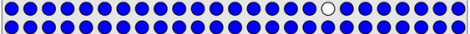

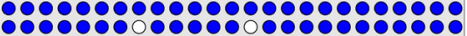

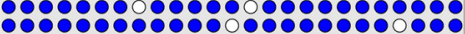

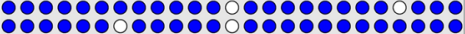

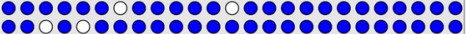

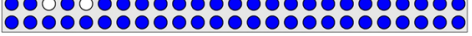
$0.90,0.99$

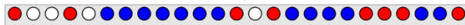
(1) (1) -

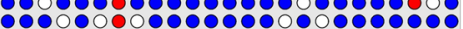
: : -

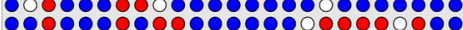

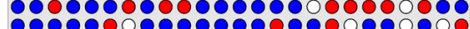

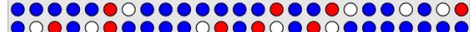

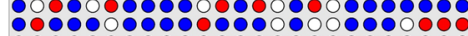

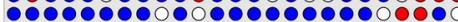

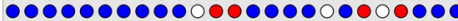

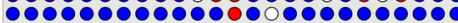

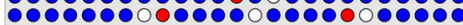

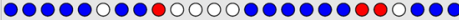

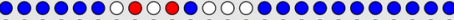

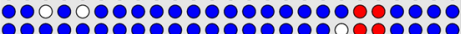

$$
0.75,0.99
$$

$00000 \bullet \bullet \bullet \bullet \bullet \bullet \bullet 000 \bullet 000000000$ 0

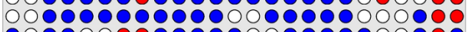
-

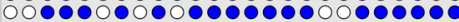
- $0 \cdot 0 \cdot 0 \cdot 0 \cdot 0 \cdot 0000000$

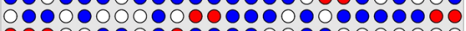

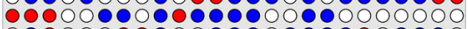
$000000000 \cdot 0 \cdot 0 \cdot 0 \cdot 00000$

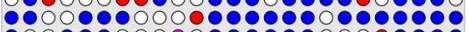
- $00000000000000000 \cdot 0$ -

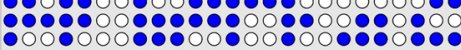
:이인 1080-00800000000 :  : :

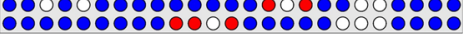

$$
0.60,0.99
$$

Fig. 11 Two-dimensional visualization of the evolution of liquid structure at the surface of a RPNM (left) and CPNM (right) for the surface saturation values of $0.90,0.75,0.60,0.45,0.30,0.15$. Empty and isolated elements are shown in white/off-white and red, respectively. Other colors indicate liquid clusters, while the main cluster is shown in blue (a single realization)

network saturation in RPNM decreases to 0.58, while the network saturation of CPNM remains nearly unchanged. This means almost all liquid is evaporated from the surface of the classical pore network during this period. Moreover, the liquid and gas elements at the 


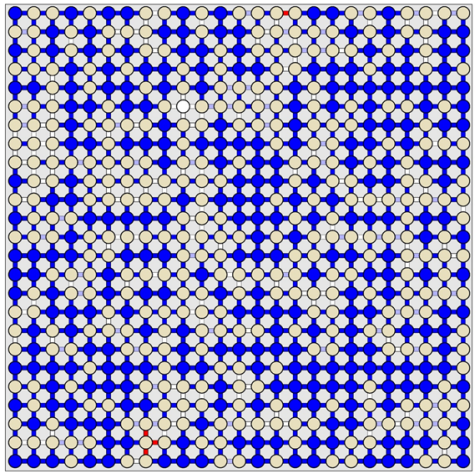

$0.45,0.44$

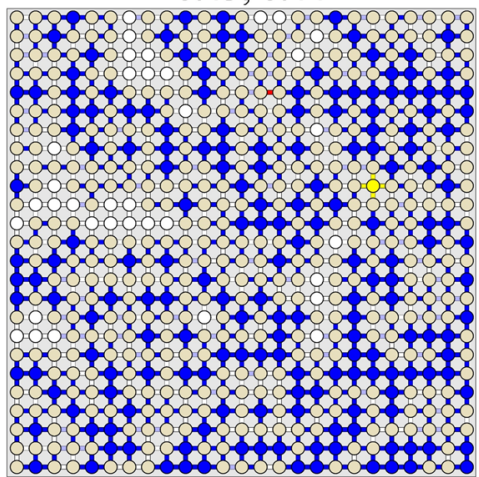

$0.30,0.32$

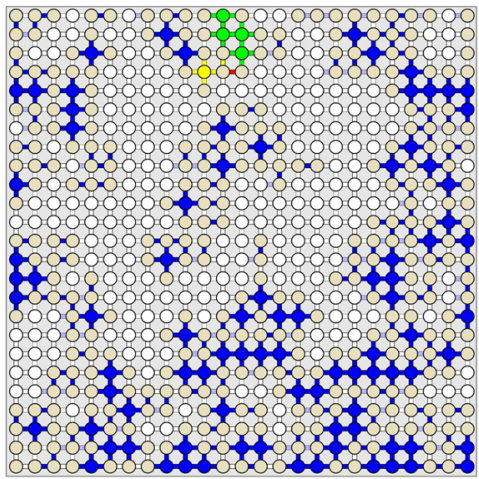

$0.15,0.24$

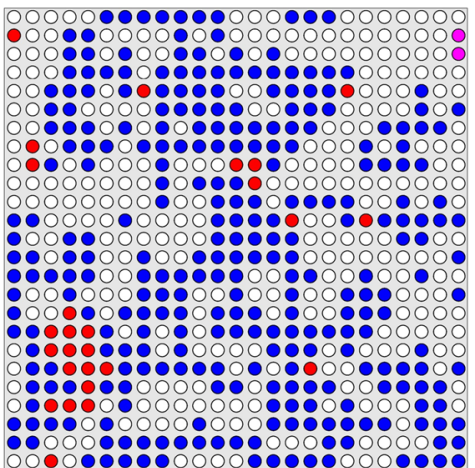

$0.45,0.97$

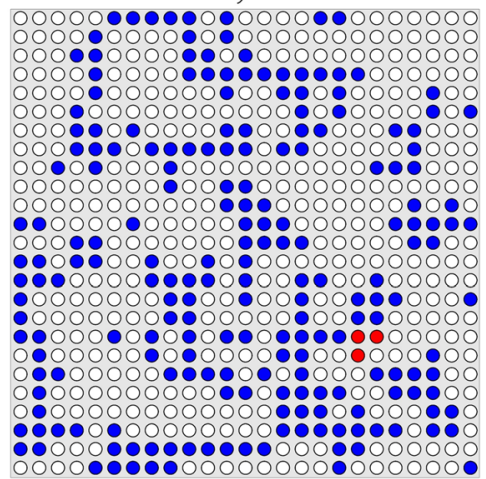

$0.30,0.82$

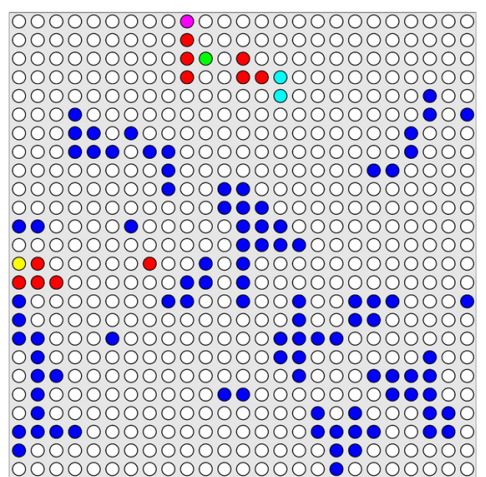

$0.15,0.72$

Fig. 11 (continued)

surface based on $\left(P_{\mathrm{v}, \mathrm{eq}}\right)$ or $\left(P_{\mathrm{v}}\right)$ are termed as wet patch and dry patch, respectively. At a lower surface saturation of 0.45 , the network saturation is 0.45 and 0.97 in RPNM and CPNM, respectively, which indicates pumping of liquid from deep inside of the network toward surface is efficient in the presence of rings. Isolated elements and different clusters at the surface represent disintegration of the liquid phase. Number of single throats at the surface of classical pore network is significant from high to low saturation (see red color 
on the right side of Fig. 11). It means single throats stay there for longer time. The number of isolated rings is insignificant in RPNM, even the number of rings is double than throats, while the number of empty elements is growing (see red and white color on the left side of Fig. 11). This indicates that the single rings dry quickly when they become isolated. Maximum liquid clusters at the surface belong to the main cluster from high to low saturation of the surface in both models (see blue color in Fig. 11).

The fact that the average vapor partial pressure at the surface of a drying capillary porous medium is lower than the equilibrium vapor partial pressure is referred to as nonlocal equilibrium effect in our previous studies (Moghaddam et al. 2017; Lu et al. 2021). This effect is decreased in RPNM as shown in Fig. 12a. Figure 12b shows standard deviation of the averaged vapor pressure at the surface for both models as a function of surface saturation. The standard deviation of the averaged vapor pressure of RPNM remains zero until low network saturation (cf. Figures $12 \mathrm{~b}$ and 4), while it increases continuously toward maximum value in CPNM. The highest standard deviation shows end of CRP/FRP transition and small part of wet patches at surface in both models. After that, surface dries out completely. The variation of the vapor pressure field due to rings also explains duration of CRP and dominance of wet patches during most of the CRP.

The evaporation rate can be fractionated into two components: the evaporation rate from the wet patches and that from the dry patches at the surface. The relative contribution of the wet and dry patches at the surface to the net evaporation rate is shown in Fig. 13. The role of dry patches in CPNM is significant and starts from the beginning of the drying process to fully dried surface. The contribution of dry patches becomes dominant at 0.25 saturation of the surface. The role of wet patches in RPNM is fully dominant from start of the drying process to surface saturation of 0.38 . After this point, dry patches start contributing but their role is still smaller than wet patches until the surface saturation reaches 0.04 . The evaporation rate is high in case of wet patches, as vapor pressure at the pore entrances is the equilibrium vapor pressure. Rings have shifted the transition between dominant contributing patches toward very low saturation and have made the wet patches sole contributor for most of the CRP.

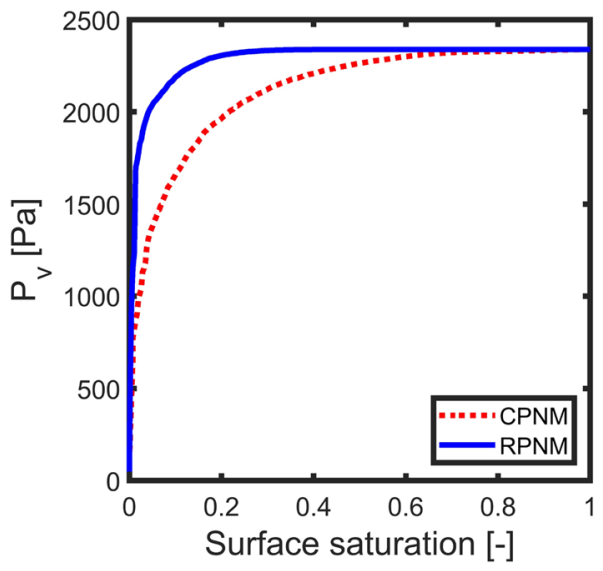

(a)

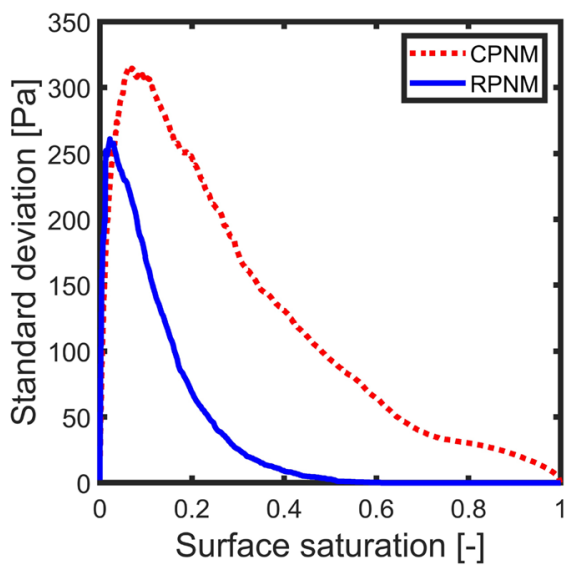

(b)

Fig. 12 Variation of the average (a) and standard deviation (b) of the vapor partial pressure as a function of surface saturation, averaged over 15 realizations 
Fig. 13 Relative contributions of dry and wet patches at the network surface to the total evaporation rate for CPNM and RPNM as a function of surface saturation, averaged over 15 realizations

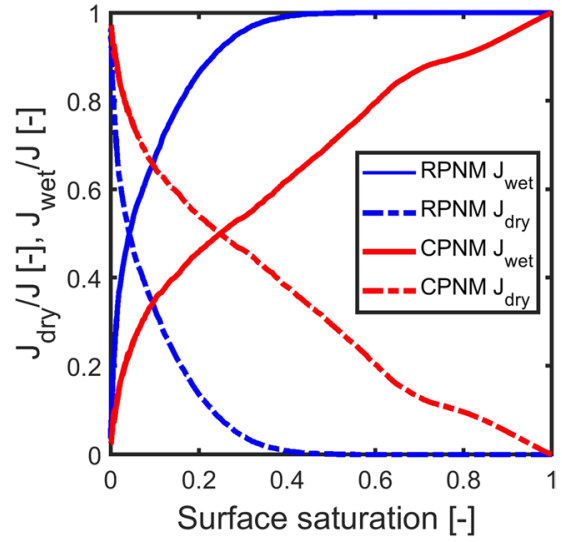

As discussed in Sect. 1, Schlünder's model provides the closure relationship of the evaporation rate with the surface wetness. This model requires three input parameters with values which need to be specified a priori. A gas diffusion length of 10L is considered in the pore network simulations, where $\mathrm{L}$ denotes the throat length. The porous medium surface of Schlünder's model is homogeneous as opposed to pore network models. The radius of a wet patch $\left(r_{t}\right)$ is taken as the mean throat radius and the surface wetness $(\theta)$ is identified from the pore network modeling as wet area of throats or surface saturation.

Figure 14 shows CRP and transition of CRP/FRP as a function of reduced saturation and the comparison between the Schlünder's model prediction and the CPNM and RPNM simulations. Reduced saturation is calculated as $\left(S_{\text {net }}-S_{\text {dry }}\right) /\left(1-S_{\text {dry }}\right)$, where $S_{\text {net }}$ and $S_{\text {dry }}$ are the network saturation and the saturation when the network surface dries, respectively. $S_{\text {dry }}$ for CPNM and RPNM is 0.64 and 0.20 , respectively. Figure 14a displays the portion of Fig. 4 which is comparable to Schlünder's model. The results obtained from the CPNM indicate a poor agreement with the Schlünder formula as shown in Fig. 14b. The agreement

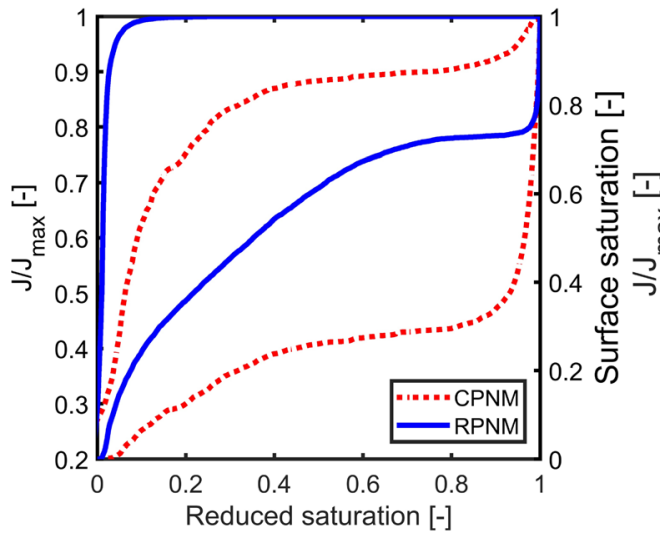

(a)

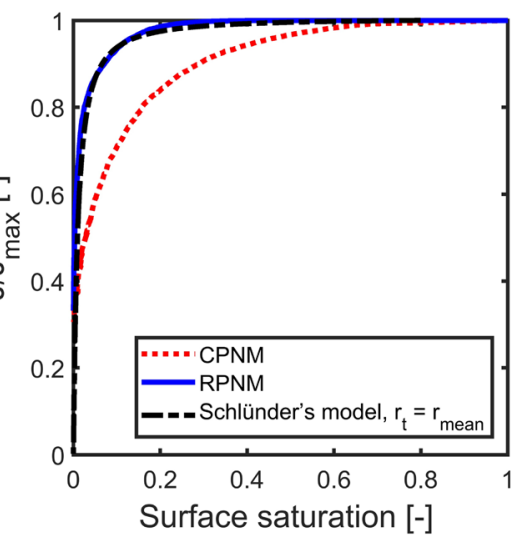

(b)

Fig. 14 a Normalized evaporation rate and surface saturation as a function of reduced network saturation and $\mathbf{b}$ normalized evaporation rate as a function of surface saturation obtained from the CPNM and RPNM simulations as well as from the solution of Schlünder's model for $r_{t}=r_{\text {mean }}$ 
is improved quite significantly with the evaporation rate based on RPNM simulations. According to Schlünder's approach, the evaporation rate from a partially saturated surface remains almost consistent with the initial evaporation rate though the surface saturation decreases that means until $\theta$ becomes small enough. The inclusion of secondary capillary structure as rings in CPNM explains the significant difference between the CPNM and Schlünder's model. The rings increase liquid connectivity and reduce the internal moisture transport resistance, promoting the surface wetness. Due to this support, the lateral vapor transfer in the boundary layer ensures that the liquid is evaporated at the initial evaporation rate. For the CPNM, the high internal moisture transport resistance prevents the replenishment of the surface wetness from the bottom of the pore network.

\section{Summary and Conclusions}

In this work, the role of discrete capillary rings in mass transfer from the surface of a slowly drying non-hygroscopic porous medium is quantified using classical pore network model (CPNM) and ring pore network model (RPNM) simulations. Results show that capillary rings support liquid transport to the medium surface by establishing saturated air conditions in drained pores even if they are not hydraulically interconnected. The results obtained from these two PNMs are compared against each other and also against that of Schlünder's model. According to the previous studies conducted using CPNMs (Moghaddam et al. 2018; Lu et al. 2021), both types of wet and dry patches at the surface contribute to the evaporation rate from the surface of the porous medium, while Schlünder's model emphasizes on the contribution of wet patches and neglects the role of dry patches. The RPNM indicates a dominant role of wet patches and minor role of dry patches at the surface toward the end of constant rate period. The findings of RPNM support relatively better the simple analytical formulation of evaporation rate as a function of extent of wet surface made by Schlünder $(1988,2004)$.

Moreover, the impact of capillary rings on drying kinetics and phase distribution is extensive. When rings are present, nearly all liquid remains belonged to the main cluster from the beginning till the end of the constant rate period during which liquid supply from the bottom toward the surface of the network is maintained. Therefore, the average vapor partial pressure at the surface stays near the equilibrium vapor pressure. Another major impact is an apparent decrease in the sharp drop observed in profiles of saturation near the evaporative surface (edge effect) and also removal of the initial transition period because of the mild drop in the surface saturation and late formation of isolated elements. Moreover, it is observed that consideration of capillary rings in PNMs increases the coordination number of pores which eventually leads to a lower irreducible saturation compared to the PNMs which exclude such secondary capillary elements (e.g., see, Moghaddam et al. 2017; Lu et al. 2020).

It would be interesting to exploit the influence of the boundary layer thickness and the spread of pore size distribution on mass transport from the porous medium surface during drying. In order to enhance the predictive capacity of such three-dimensional ring PNMs, viscous effects both in the liquid films and in the bulk liquid clusters should also be taken into account.

Acknowledgements The partial financial support for this work by the Higher Education Commission (HEC), Pakistan, is gratefully acknowledged. 
Funding Open Access funding enabled and organized by Projekt DEAL.

Open Access This article is licensed under a Creative Commons Attribution 4.0 International License, which permits use, sharing, adaptation, distribution and reproduction in any medium or format, as long as you give appropriate credit to the original author(s) and the source, provide a link to the Creative Commons licence, and indicate if changes were made. The images or other third party material in this article are included in the article's Creative Commons licence, unless indicated otherwise in a credit line to the material. If material is not included in the article's Creative Commons licence and your intended use is not permitted by statutory regulation or exceeds the permitted use, you will need to obtain permission directly from the copyright holder. To view a copy of this licence, visit http://creativecommons.org/licenses/by/4.0/.

\section{References}

Chauvet, F., Duru, P., Geoffroy, S., Prat, M.: Three periods of drying of a single square capillary tube. Phys. Rev. Lett. 103, 124502 (2009)

Chauvet, F., Duru, P., Prat, M.: Depinning of evaporating liquid films in square capillary tubes: Influence of corners roundness. Phys. Fluids 22, 112113 (2010)

Chen, C., Duru, P., Joseph, P., Geoffroy, S., Prat, M.: Control of evaporation by geometry in capillary structures. From confined pillar arrays in a gap radial gradient to phyllotaxy-inspired geometry. Sci. Rep. 7, 015110 (2017)

Chen, C., Joseph, P., Geoffroy, S., Prat, M., Duru, P.: Evaporation with the formation of chains of liquid bridges. J. Fluid Mech. 837, 703-728 (2018)

Daian, J.F., Saliba, J.: Determining a representative random pore-network for moisture sorption and migration in cement mortar. Int. J. Heat Mass Transf. 34, 2081-2096 (1991)

Dillard, L.A., Blunt, M.J.: Development of a pore network simulation model to study nonaqueous phase liquid distribution. Water Resour. Res. 36(2), 439-454 (2000)

Faure, P., Coussot, P.: Drying of a model soil. Phys. Rev. E 82, 036303 (2010)

Kharaghani, A., Metzger, T., Tsotsas, E.: An irregular pore network model for convective drying and resulting damage of particle aggregates. Chem. Eng. Sci. 75, 267-278 (2012)

Kharaghani, A., Mahmood, H.T., Wang, Y.J., Tsotsas, E.: Three-dimensional visualization and modelling of liquid film rings observed during drying of random particle packings. Int. J. Heat Mass Transf. 177, 121505 (2021)

Laurindo, J.B., Prat, M.: Numerical and experimental network study of evaporation in capillary porous media. Phase Distributions. Chem. Eng. Sci. 51, 5171-5185 (1996)

Laurindo, J.B., Prat, M.: Numerical and experimental network study of evaporation in capillary porous media. Drying Rates. Chem. Eng. Sci. 53, 2257-2269 (1998)

Lehmann, P., Or, D.: Effect of wetness patchiness on evaporation dynamics from drying porous surfaces. Water Resour. Res. 49, 8250-8262 (2013)

Lenormand, R., Touboul, E., Zarcone, C.: Numerical models and experiments on immiscible displacement in porous media. J. Fluid Mech. 189, 165-187 (1988)

Lu, X., Kharaghani, A., Tsotsas, E.: Transport parameters of macroscopic continuum model determined from discrete pore network simulations of drying porous media: Throat-node vs. throat-pore configurations. Chem. Eng. Sci. 223, 115723 (2020)

Lu, X., Tsotsas, E., Kharaghani, A.: Insights into evaporation from the surface of capillary porous media gained by discrete pore network simulations. Int. J. Heat Mass Transf. 168, 120877 (2021)

Luikov, A.V.: Heat and mass transfer in capillary-porous bodies. Pergamon Press, Oxford (1966)

Metzger, T., Tsotsas, E., Prat, M.: Pore-network models: a powerful tool to study drying at the pore level and understand the influence of structure on drying kinetics. In: Tsotsas, E., Mujumdar, A.S. (eds.) Modern Drying Technology, Vol. 1: Computational Tools at Different Scales, pp. 57-102. Wiley$\mathrm{VCH}$, Weinheim (2007a)

Metzger, T., Irawan, A., Tsotsas, E.: Influence of pore structure on drying kinetics: a pore network study. AIChE J. 53(12), 3029-3041 (2007b)

Moghaddam, A.A., Kharaghani, A., Tsotsas, E., Prat, M.: Kinematics in a slowly drying porous medium: reconciliation of pore network simulations and continuum modeling. Phys. Fluids 29, 022102 (2017)

Moghaddam, A.A., Kharaghani, A., Tsotsas, E., Prat, M.: A pore network study of evaporation from the surface of a drying non-hygroscopic porous medium. AIChE J. 64(4), 1435-1447 (2018) 
Nowicki, S.C., Davis, H.T., Scriven, L.E.: Microscopic determination of transport parameters in drying porous media. Dry. Technol. 10, 926-946 (1992)

Philip, J.R., De. Vries, D.: Moisture movement in porous materials under temperature gradients. Trans. Am. Geophys. Union 38, 222-232 (1957)

Prat, M.: Percolation model of drying under isothermal conditions in porous media. Int. J. Multiph. Flow 19, 691-704 (1993)

Sahimi, M., Hughes, B.D., Scriven, L.E., Ted Davis, H.: Dispersion in flow through porous media-I. OnePhase Flow. Chem. Eng. Sci. 41, 2103-2122 (1986)

Schlünder, E.U.: On the mechanism of the constant drying rate period and its relevance to diffusion controlled catalytic gas phase reactions. Chem. Eng. Sci. 43, 2685-2688 (1988)

Schlünder, E.U.: Drying of porous material during the constant and the falling rate period: a critical review of existing hypotheses. Dry. Technol. 22, 1517-1532 (2004)

Surasani, V.K., Metzger, T., Tsotsas, E.: Consideration of heat transfer in pore network modelling of convective drying. Int. J. Heat Mass Transf. 51, 2506-2518 (2008)

Talbi, M., Prat, M.: About Schlünder's model: a numerical study of evaporation from partially wet surfaces. Dry. Technol. 37, 513-524 (2019)

Wilkinson, D., Willemsen, J.F.: Invasion percolation: a new form of percolation theory. J. Phys. A 16, 33653376 (1983)

Whitaker, S.: Coupled transport in multiphase systems: a theory of drying. Adv. Heat Transf. 31, 1-104 (1998)

Wang, Y.J., Kharaghani, A., Metzger, T., Tsotsas, E.: Pore network drying model for particle aggregates: Assessment by X-Ray microtomography. Dry. Technol. 30, 1800-1809 (2012)

Wu, R., Kharaghani, A., Tsotsas, E.: Capillary valve effect during slow drying of porous media. Int. J. Heat Mass Transf. 94, 81-86 (2016)

Wu, R., Zhao, C.Y., Tsotsas, E., Kharaghani, A.: Convective drying in thin hydrophobic porous media. Int. J. Heat Mass Transf. 112, 630-642 (2017)

Vorhauer, N., Wang, Y.J., Kharaghani, A., Tsotsas, E., Prat, M.: Drying with formation of capillary rings in a model porous medium. Transport Porous Med. 110, 197-223 (2015)

Yiotis, A.G., Boudouvis, A.G., Stubos, A.K., Tsimpanogiannis, I.N., Yortsos, Y.C.: Effect of liquid films on the drying of porous media. AIChE J. 50, 2721-2737 (2004)

Yiotis, A.G., Tsimpanogiannis, I.N., Stubos, A.K., Yortsos, Y.C.: Pore-network study of the characteristic periods in the drying of porous materials. J. Colloid Interface Sci. 297, 738-748 (2006)

Yiotis, A.G., Tsimpanogiannis, I.N., Stubos, A.K., Yortsos, Y.C.: Coupling between external and internal mass transfer during drying of a porous medium. Water Resour. Res. 43, W06403 (2007)

Yiotis, A.G., Salin, D., Tajer, E.S., Yortsos, Y.C.: Analytical solutions of drying in porous media for gravitystabilized fronts. Phys. Rev. E 85, 046308 (2012)

Publisher's Note Springer Nature remains neutral with regard to jurisdictional claims in published maps and institutional affiliations. 